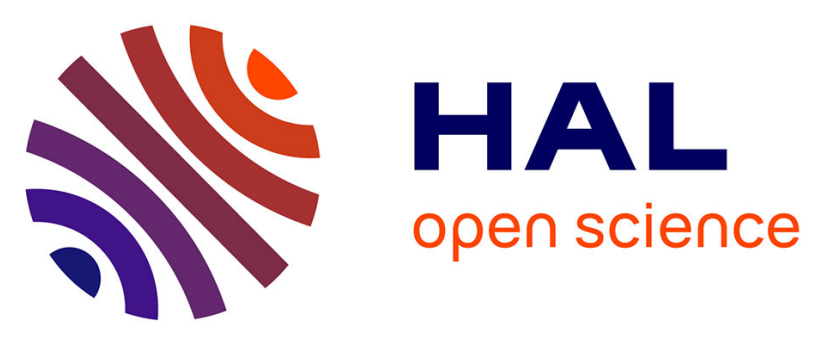

\title{
A practical online time-varying delay estimation of remote control system based on adaptive super-twisting algorithm
}

Yang Deng, Vincent Léchappé, Sébastien Rouquet, Emmanuel Moulay, Franck Plestan

\section{To cite this version:}

Yang Deng, Vincent Léchappé, Sébastien Rouquet, Emmanuel Moulay, Franck Plestan. A practical online time-varying delay estimation of remote control system based on adaptive super-twisting algorithm. Proceedings of the 4th Conference on Control Techology and Applications (CCTA), Aug 2020, Montreal, Canada. 10.1109/CCTA41146.2020.9206286 . hal-02928666

\section{HAL Id: hal-02928666 \\ https://hal.science/hal-02928666}

Submitted on 2 Sep 2020

HAL is a multi-disciplinary open access archive for the deposit and dissemination of scientific research documents, whether they are published or not. The documents may come from teaching and research institutions in France or abroad, or from public or private research centers.
L'archive ouverte pluridisciplinaire HAL, est destinée au dépôt et à la diffusion de documents scientifiques de niveau recherche, publiés ou non, émanant des établissements d'enseignement et de recherche français ou étrangers, des laboratoires publics ou privés. 


\title{
A practical online time-varying delay estimation of remote control system based on adaptive super-twisting algorithm
}

\author{
Yang Deng ${ }^{1}$, Vincent Léchappé ${ }^{2}$, Sébastien Rouquet ${ }^{1}$, Emmanuel Moulay $^{3}$ and Franck Plestan ${ }^{1}$
}

\begin{abstract}
This article deals with the practical online delay estimation of the remote control system by using an external signal. The external signal can be considered as a special communication loop of the remote control system, and it requires no information from the control system. The delay estimator is based on the adaptive super-twisting algorithm, it accurately estimates the time-varying delay with self-tuned parameters. Moreover, the proposed method also attenuates the effect of the channel inherent noise and deception attack via the network. Finally, this method is validated on a WiFi communication network, and some simulations are given to illustrate the performance and the robustness of the proposed method.
\end{abstract}

Keywords-Delay estimation, time-varying delay, adaptive super-twisting algorithm.

\section{INTRODUCTION}

Remote-control technique [1] is widely used in engineering thanks to the development of telecommunication networks. In such systems, the measurement and the control signal can be transmitted via wireless communication. However, one must consider the time-delay introduced by the data transmission since the time-delay is a source of instability [2] of the control system. Predictor-feedback (predictor-based controller) [3], [4] is an effective way to compensate the time-delay if the delay value is known. If the remote-control system (RCS) is subject to unknown time-delay, then one can firstly estimate the time-delay and then stabilize the RCS with a predictor-based controller. Thus, time-delay estimation (TDE) is significant to get effective control of the RCS with unknown time-delay.

\section{A. State of the art of TDE method}

Many works deal with TDE techniques in the past few decades. In [5] and [6], the uncertain parameters and timedelays of linear TDS are estimated with least-square algorithm and convolution-based algebraic approach, respectively. In [7] and [8], adaptive techniques are proposed to estimate the time-delay of continuous and sampled-data linear systems, but both of them are sensitive to the parameter and

\footnotetext{
${ }^{1}$ Yang Deng, Sébastien Rouquet and Franck Plestan are with Ecole Centrale de Nantes, LS2N UMR CNRS 6004, Nantes, France. E-mail addresses: yang.deng@ec-nantes.fr, sebastien.rouquet@ec-nantes.fr,

${ }^{2}$ Vincent Léchappé is with Univ Lyon, INSA Lyon, ECL, Université Claude Bernard Lyon 1, CNRS, Ampère, F-69621, Villeurbanne, France. E-mail address: vincent. lechappeeinsa-lyon. fr

${ }^{3}$ Emmanuel Moulay is with Université de Poitiers, XLIM UMR CNRS 7252, Poitiers, France. E-mail address: emmanuel moulayduniv-poitiers.fr
}

the initial condition of the adaptation law. The sliding mode method is involved in [9] to identify the state delay of a class of nonlinear systems with local exponential convergence. The authors of [10] propose an optimal delay estimator that minimizes a cost function based on the delay estimation error.

All of the previously cited papers are dedicated to constant TDE problem, whereas the following references tackle the time-varying TDE problem. The authors of [11] firstly provide a sliding mode observer to identify the time-varying delay of linear systems, but the initial condition of the delay estimator has to be sufficiently close to the unknown time-delay. In [12], an online neural network-based TDE algorithm is proposed to deal with the time-varying delay of nonlinear systems. However, the estimation error cannot accurately tend towards zero and the computational load is heavy if the neural network is not well trained. The authors of [13] provide an adaptive method that estimates slow-varying or piecewise-constant time-delays with slight fluctuations. The majority of the references mentioned above ([5], [6], [9], [10], [11]) requires the delay identifiability [14] of the system. Several methods ([8], [12]) have high computation costs, so they cannot be easily implemented on a real system. Moreover, it is observed that the TDE problem for fastvarying delay remains challenging to the existing methods.

\section{B. Practical TDE solution of $R C S$}

In order to stabilize a RCS with time-varying delay, the authors of [15] propose a practical TDE solution: the controller sends the system clock to the plant, a digital signal processor (DSP) implemented on the plant receives the system clock information and sends it back to the controller, then the round-trip delay is measured by taking the difference between the sending time and the receiving time of the clock. The main advantages of [15] are:

- the TDE algorithm is isolated from the control system since the system clock is isolated from the system, it does not require any information or property of the control system like linearity or delay identifiability defined in [14];

- the TDE algorithm works with low computation cost, it is more efficient than the neural network based approach [12];

- all computations are running on the same node (i.e. the controller node), no clock synchronization is required.

Inspired by the work of [15], a super-twisting (STW) algorithm based TDE technique is introduced in [16] with 
experimental results. By comparing with [15], the following improvements can be found in [16]:

- in [15], the system clock $(s(t)=t)$ should be used to estimate the round-trip delay, but [16] works with all monotonic signals (including system clock);

- [16] is more robust than [15] with respect to the "channel inherent noise" (i.e. the noise or perturbation in the communication channel or at the receiving terminal [17]).

\section{Main contributions of this paper}

This work is the continuation of the paper [16], the adaptive super-twisting algorithm (ASTW) [18] is used to replace the standard super-twisting algorithm. As stated in [16, Remark 1], the estimation performance depends on the parameter tuning, unsuited parameter leads to undesirable chattering [19, p.6-8] or degrades the TDE performance. The above drawback is overcome with the adaptive supertwisting algorithm, and the parameters are self-adapted (i.e. be the smallest value that ensures the predefined estimation accuracy). The above discussions are illustrated by the experimental results given in subsection IV-A.

In [16], it is shown that the super-twisting algorithm based TDE method is more robust than the measurement approach [15] with respect to the channel inherent noise. With the proposed method, this advantage is preserved. Moreover, the simulation results show that the proposed method is also more robust than [15] when the communication loop is subject to deception attacks [20, Section VI].

\section{Organisations}

This paper is organized as follows. Some notations and the problem statement are addressed in Section II. The main results of this paper are introduced in Section III. The performance of the proposed method is illustrated by the experiment and simulation results given in Section IV. Finally, the conclusion and future works are given in Section V.

\section{Preliminaries}

This section includes the mathematical notations, the problem statement and the estimation architecture of the proposed method.

\section{A. Notations}

In this paper, the following notations are used. Let $\mathbf{C}^{k}$ denote the set of all functions with $k$ times continuous derivatives. The $\mathscr{L}_{2}$ norm of an integrable signal is defined as $\|\cdot\|$. The right-hand time-derivative of a function $f(\cdot)$ at instant $t-h(t)$ reads as $\dot{f}(t-h(t))$, and $\frac{d}{d t}(f(t-h(t)))$ denotes the right-hand time-derivative of the function $t \mapsto$ $f(t-h(t))$. Recall the chain rule given in [21, Theorem 5.5], the relation between $\frac{d}{d t} f(t-h(t))$ and $\dot{f}(t-h(t))$ is given as

$$
\frac{d}{d t} f(t-h(t))=\dot{f}(t-h(t))(1-\dot{h}(t)) .
$$

The sign-function [19, eqs. (1.13)-(1.14)] satisfies

$$
\operatorname{sign}(x)=\left\{\begin{array}{l}
1, \quad \text { if } \quad x>0 \\
-1, \text { if } x<0
\end{array}\right.
$$

and $\operatorname{sign}(0) \in[-1,1]$.

\section{B. Estimation scheme and problem statements}

Consider a remote data transmission (RDT) process between two nodes (Nodes 1 and 2) with time-varying delays due to communication lag or communication congestion. The architecture of the RDT process is presented in Figure 1. The input and output delays are respectively denoted as $h_{i}(t)$

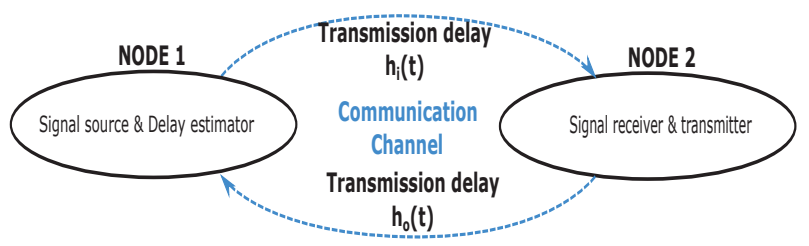

Fig. 1: Architecture of the remote data transmission (reprint from [16, Fig. 2]).

and $h_{o}(t)$. As a consequence, the round-trip delay reads as

$$
h(t)=h_{o}(t)+h_{i}\left(t-h_{o}(t)\right) \text {. }
$$

If the time-delays are constant, (3) can be simplified as $h=h_{i}+h_{o}$. One assumes that an external signal $s(t)(e . g$. system clock in [15]) is transmitted between the two nodes mentioned in Figure 1. The delay estimator implemented on Node 1 generates the external signal $s(t)$ and sends it to Node 2. After receiving the delayed signal $s\left(t-h_{i}(t)\right)$, Node 2 next sends the delayed signal back to the delay estimator. Because of the output-delay $h_{o}(t)$, the delay estimator finally receives $s(t-h(t))$.

Remark 1: The external signal $s(t)$ is totally defined by the user and is independent of the control system. It can be regarded as a special communication loop to provide the online estimation of the round-trip transmission delay (3). For instance, the delay measurement technique [15] uses the system clock to measure the round-trip delay, so it is possible to say that [15] chooses an external signal $s(t)=t$ to estimate the time-delay.

The problem to solve is the design of a delay estimator $\hat{h}(t)$ that globally converges to the round-trip delay $h(t)$ by using the knowledge of $s(t)$ and $s(t-h(t))$.

Remark 2: The proposed method can be used for the control of RCS [1, Chapter 3.4] with unknown transmission delays. As shown in Figure 2, the delay estimator sends the external signal $s(t)$ along with the control input $u(t)$ and receives the delayed external signal $s(t-h(t))$ with the system output $y(t)$ by using the same communication channel. Remind that the black arrows of Figure 2 represent the control loop and the green arrows represent the delay estimation loop. The round-trip delay of the remote control system is estimated only by the estimation loop $(s(t)$ and $s(t-h(t)))$, and the delay estimation is transmitted to the control loop in order to stabilize the system. In [4], the 


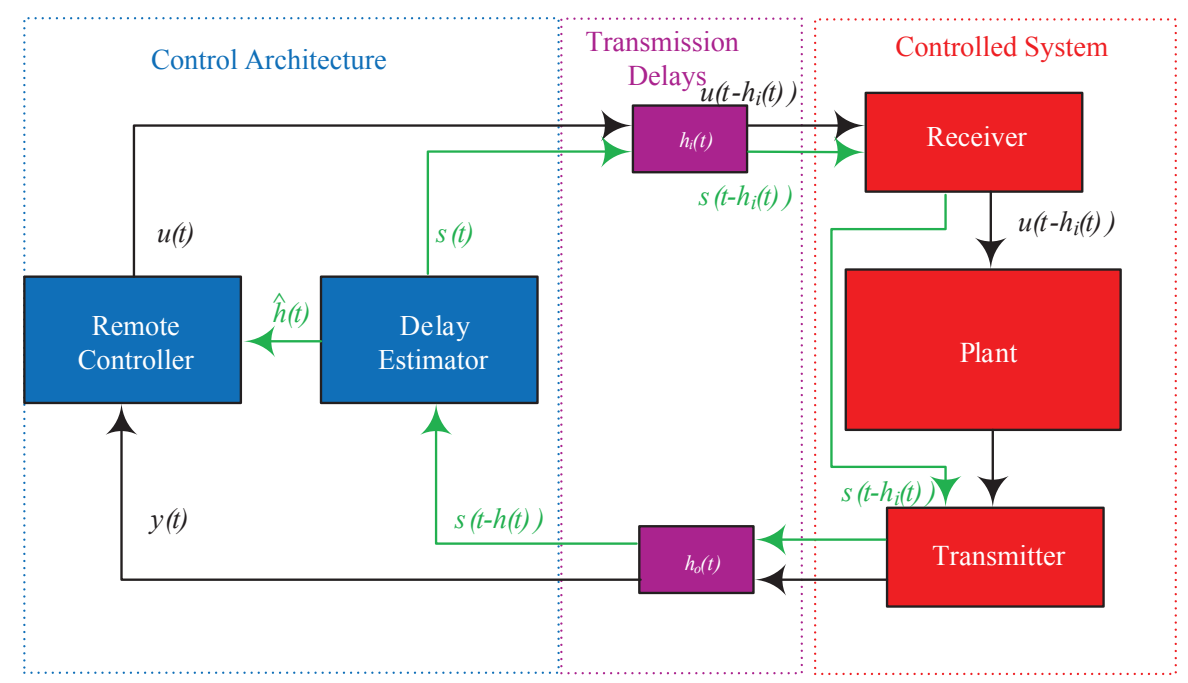

Fig. 2: Delay estimation scheme of remote control system by using external signal (reprint from [16, Fig. 1]).

stabilization of linear time-invariant (LTI) systems with input and output time-varying delays is studied, the statements in $[4$, p.27] explain that one can use the round-trip delay (3) to build a predictor-feedback control law.

\section{ADAPTIVE SUPER-TWISTING ALGORITHM BASED TDE METHOD}

In this section, a delay estimator based on adaptive supertwisting algorithm is proposed. Some assumptions and definitions are stated in subsection III-A. The main results are given in subsection III-B.

\section{A. Assumptions and definitions}

Denote the external signal $s(t)$ and the unknown timevarying delay $h(t)$ which is upper bounded by $h_{\max }$. The following two assumptions are introduced.

Assumption 1: The external signal $s(t) \in \mathbf{C}^{2}$ satisfies that

$$
\underline{\varepsilon} \leq|\dot{s}(t)| \leq \bar{\varepsilon}, \quad t \geq-h_{\max },
$$

and

$$
|\ddot{s}(t)| \leq \varepsilon^{\prime}, \quad t \geq-h_{\max } .
$$

The positive constants $\underline{\varepsilon}, \bar{\varepsilon}$, and $\varepsilon^{\prime}$ are assumed to be known.

Assumption 1 implies that the external signal $s(t)$ is strictly monotonic.

Remark 3: In [9] and [11], the delay-identifiability of the TDS is required. Indeed, Assumption 1 shows that $s(t)$ is globally identifiable in the sense of the delay identifiability theory [9, p.268, Definition 2]. However, as stated in Remark 1, this signal $s(t)$ is generated by the user and it is independent of the control system. In other words, with the proposed approach, the round-trip delay is estimated by using a specific delay-identifiable signal, but not the system information. Thus, the delay identifiability of the control system can be relaxed by using the proposed technique.
Assumption 2: The first and second derivative of $h(t)$ are bounded such that

$$
|\dot{h}(t)| \leq \delta, \quad t \geq-h_{\max }
$$

and

$$
|\ddot{h}(t)| \leq \delta^{\prime}, \quad t \geq-h_{\max } .
$$

The bounds $\delta$ and $\delta^{\prime}$ are not assumed to be known.

As mentioned in [16, Section III-A], Assumption 2 can be verified in application, for instance, consider a time-varying delay $h(t)$ that is upper bounded by $h_{\max }$. It is possible to find the bounds

$$
\delta \leq \frac{h_{\max }}{T_{S}}
$$

and

$$
\delta^{\prime} \leq \frac{\frac{h_{\max }}{T_{s}}-\left(-\frac{h_{\max }}{T_{s}}\right)}{T_{s}}=\frac{2 h_{\max }}{T_{s}^{2}},
$$

with $T_{s}$ the sampling period of the RDT process. By using the worst case analysis, if $h(t)$ varies from $h_{\max }$ to 0 in one sampling period, then its derivative must be upper bounded by (8), and (9) can be ensured in the same way.

After stating the necessary assumptions, the definition of the "real sliding mode" [18, p.761] is recalled in the sequel.

Definition 1: Given the sliding variable $\sigma(s, t)$, the "2-real sliding mode" of the variable $s$ is defined as

$$
\mathscr{S}^{*}=\left\{s:|\sigma(s, t)| \leq \eta_{1}, \quad|\dot{\sigma}(s, t)| \leq \eta_{2}\right\} .
$$

with $\eta_{1}, \eta_{2}>0$.

\section{B. Main results}

Before stating the main results of this work, one first recalls the main result of the previous work [16].

Theorem 1: [16] Consider the TDE problem formulated in Section II-B, suppose that the external signal $s(t)$ satisfies 
Assumption 1, and the time-varying delay $h(t)$ satisfies Assumption 2. Define the sliding variable

$$
\sigma(t)=s(t-\hat{h}(t))-s(t-h(t))
$$

The following dynamics

$$
\left\{\begin{array}{l}
\dot{\hat{h}}(t)=1-\frac{1}{\dot{s}(t-\hat{h}(t))} w(t) \\
w(t)=-\lambda|\sigma(t)|^{1 / 2} \operatorname{sign}(\sigma(t))+w_{1}(t), \\
\dot{w}_{1}(t)=-\alpha \cdot \operatorname{sign}(\sigma(t))
\end{array}\right.
$$

ensure the finite-time delay estimation

$$
\hat{h}(t)=h(t), \quad t \geq t_{F}
$$

if the two parameters $\lambda, \alpha$ are sufficiently large such that

$$
\begin{aligned}
& \alpha \geq C=\bar{\varepsilon} \delta^{\prime}+\varepsilon^{\prime}(1+\delta)^{2}, \\
& \lambda^{2} \geq 4 C \frac{\alpha+C}{\alpha-C} .
\end{aligned}
$$

In real application, the $\mathrm{TDE}^{C}$ performance of Theorem 1 totally depends on the choice of the parameters $\alpha$ and $\lambda$ (namely, the bounds $\delta$ and $\delta^{\prime}$ ). If the parameters are too small, then the delay estimator fails to converge, and if the parameters are too large, they result in undesirable chattering [19, p.6-8]. In order to deal with the case when the bounds $\delta$ and $\delta^{\prime}$ are not perfectly known beforehand, the main result of this paper is proposed.

Theorem 2: Consider the TDE problem formulated in Section II-B, suppose that the external signal $s(t)$ satisfies Assumption 1, the time-varying delay $h(t)$ satisfies Assumption 2 , and the sliding variable (11). Given the TDE dynamics

$$
\left\{\begin{array}{l}
\dot{\hat{h}}(t)=1-\frac{1}{\dot{s}(t-\hat{h}(t))} w(t), \\
w(t)=-\lambda(t)|\sigma(t)|^{1 / 2} \operatorname{sign}(\sigma(t))+w_{1}(t), \\
\dot{w}_{1}(t)=-\frac{\alpha(t)}{2} \cdot \operatorname{sign}(\sigma(t)),
\end{array}\right.
$$

with the adaptive parameters

$$
\begin{aligned}
& \dot{\lambda}(t)= \begin{cases}\omega_{1} \sqrt{\frac{\gamma_{1}}{2}} \operatorname{sign}(|\sigma(t)|-\mu), & \text { if } \lambda(t)>\lambda_{m} \\
\eta, & \text { if } \lambda(t) \leq \lambda_{m}\end{cases} \\
& \alpha(t)=2 \zeta \lambda(t)
\end{aligned}
$$

where $\zeta>0, \omega_{1}>0,1>\gamma_{1}>0, \eta>0$ are arbitrarily chosen, and $\lambda_{m}>0$ is sufficient small. If $\lambda(0)>\lambda_{m}$, then there exist $\eta_{1}>\mu, \eta_{2}>0$, and $t_{F}>0$ such that the 2-real sliding mode (10) is established for all $t \geq t_{F}$. Finally, the delay estimation error $e(t)=h(t)-\hat{h}(t)$ satisfies that

$$
|e(t)| \leq \frac{\eta_{1}}{\varepsilon}, \quad t \geq t_{F}
$$

Proof: Taking the time-derivative of the sliding variable (11) leads to

$$
\dot{\sigma}(t)=\dot{s}(t-\hat{h}(t))-\frac{d}{d t} s(t-h(t))-\dot{s}(t-\hat{h}(t)) \dot{\hat{h}}(t) .
$$

By virtue of Assumption 1 , one has $\dot{s}(t-\hat{h}(t)) \neq 0$ for all $t \geq 0$, then it is possible to substitute the first dynamic of (15) into (18) without singularity. Therefore, (18) is equivalent to the following dynamic:

$$
\dot{\sigma}(t)=a(t)+b w(t)
$$

with $a(t)=\frac{d}{d t} s(t-h(t))$ and $b=1$. Indeed, due to Assumptions $1-2$, the term $a(t)$ has bounded derivative such that

$$
\begin{aligned}
|\dot{a}(t)| & =\left|\dot{s}(t-h(t)) \ddot{h}(t)-\ddot{s}(t-h(t))(1-\dot{h}(t))^{2}\right| \\
& \leq \bar{\varepsilon} \delta^{\prime}+\varepsilon^{\prime}(1+\delta)^{2} .
\end{aligned}
$$

In (19), since $a(t)$ has bounded derivative and $b$ is constant, then the dynamic (19) satisfies all of the 5 assumptions given in [18, p.760,A1-A5]. Finally, according to [18, Theorem 1], the super-twisting algorithm (15) with adaptive parameters (16) establishes the 2-real sliding mode (10).

Next, one derives the estimation error bound (17) from the 2-real sliding mode (10). Apply the mean value theorem [21, Theorem 5.11] to (11), there exists $h_{1} \in$ $[\min \{(h(t)), \hat{h}(t)\}, \max \{(h(t)), \hat{h}(t)\}]$ such that

$$
\begin{aligned}
|\sigma(t)| & =\left|\dot{s}\left(t-h_{1}\right)\right||t-\hat{h}(t)-(t-h(t))| \\
& =\left|\dot{s}\left(t-h_{1}\right)\right||e(t)| .
\end{aligned}
$$

Thus, with the use of Assumption 1, the estimation error satisfies that

$$
|e(t)|=\frac{|\sigma(t)|}{\left|\dot{s}\left(t-h_{1}\right)\right|} \leq \frac{|\sigma(t)|}{\underline{\varepsilon}} .
$$

Finally, the main result (17) can be obtained by combining (22) and (10).

Theorem 2 presents an adaptive super-twisting algorithm based TDE technique and it has the following properties:

- the parameter tuning (14) is no longer required, it is replaced by the adaptive gains (16);

- the delay estimation $\hat{h}(t)$ cannot reach the ideal finitetime convergence (13), it can only establish the 2real sliding mode (10). However, one can improve the estimation accuracy by tuning the parameter $\mu$ in (16) smaller.

\section{ILLUSTRATIVE EXAMPLES}

In this section, 3 examples are given to illustrate the proposed method by considering TDE of the following round-trip delay

$$
h(t)=1.3+0.3 \sin (3 t)+0.5 \sin (2(t-0.8-0.3 \sin (3 t)))
$$

that is introduced by the following input and output delays:

$$
\begin{aligned}
& h_{i}(t)=0.5+0.5 \sin (2 t), \\
& h_{o}(t)=0.8+0.3 \sin (3 t) .
\end{aligned}
$$

The first example is an experiment that compares the performances of the proposed method and the one of [16]. In the second example, a simulation is given to illustrate that the proposed method provides better performance than [15] in the presence of channel inherent noise. Moreover, the proposed method is also better than [15] when the communication loop is subject to deception attack, this property is shown by the simulation results provided by the third example. 


\section{A. Experiment: comparison with [16]}

In this subsection, the proposed method is implemented on the test bench of [16] and compared with the standard super-twisting method (12). The test bench is based on the communication between two computers through WiFi network, see Figure 3.

Indeed, the time-delays $h_{r i}(t), h_{r o}(t)$ (see Figure 3) intro-

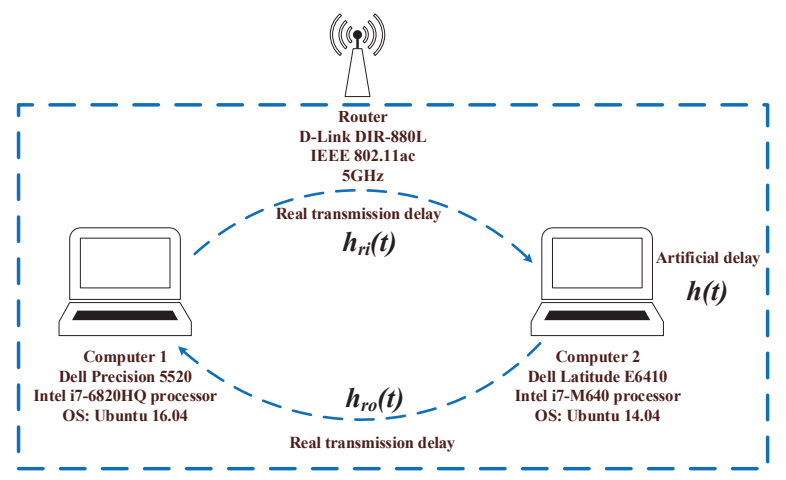

Fig. 3: Test bench based on the communication between two computers via WiFi network.

duced by a real network are small (about several milliseconds) and in a random manner, which are different from the ones given in (24). In order to create the transmission delay (23) on the test bench, an artificial delay equals to $h(t)$ is added onto Computer 2. As a consequence, the real roundtrip delay of this experiment is a composite function of the artificial delay and the real input/ output delays introduced by the network, it can be approximated as

$$
h_{\text {approx }}(t)=h_{r i}(t)+h(t)+h_{r o}(t)
$$

since $h_{r i}(t)$ and $h_{r o}(t)$ are much smaller than $h(t)$.

In the experiment, the external signal is chosen as $s(t)=t$, and the two algorithms are tested with sampling time $T_{s}=$ $0.005 \mathrm{~s}$. The parameters of the proposed method are set to $\omega_{1}=1.1, \gamma_{1}=0.8, \mu=0.0025, \lambda_{m}=0.1, \eta=0.3, \zeta=0.7$, and $\lambda(0)=9$. The method of [16] is tested with two cases, the first case is with $\lambda=5$ and $\alpha=7.5$, the second case is based on $\lambda=50$ and $\alpha=15$.

The experimental results are presented in Figure 4. Firstly, Figures $4 \mathrm{a}-4 \mathrm{~b}$ show that the round-trip delay is estimated by the proposed method, and the adaptive gain $\lambda(t)$ is decreased to a suitable size (the gain $\alpha(t)$ is not given because it is proportional to $\lambda(t)$, see (16)). Figure $4 \mathrm{c}$ displays the approximated estimation error $e_{\text {approx }}(t)=h_{\text {approx }}(t)-\hat{h}(t)$ of the 3 cases, it is observed that the method (12) with $\lambda=50$ and $\alpha=15$ brings high-amplitude chattering, and the chattering is reduced if one uses lower gains $\lambda=5, \alpha=7.5$. However, it is impossible to find the best tuning parameters beforehand, then the proposed method is used to handle this problem. See Figure 4c, the proposed method ensures the similar estimation accuracy with (12) $(\lambda=5, \alpha=7.5)$ without any prior knowledge of the parameters. Thus, the experimental results given in Figure 4 highlight the use of

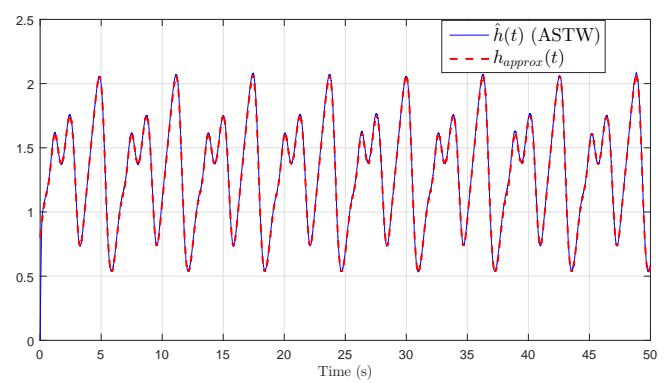

(a) Time-varying delay $h(t)$ and delay estimation $\hat{h}(t)$ with the proposed method (Experiment).

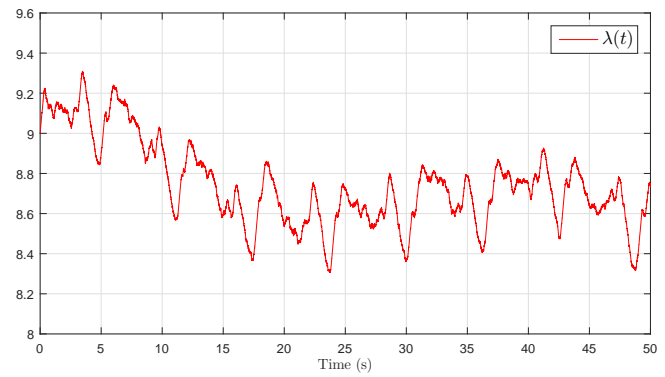

(b) Adaptive gain $\lambda(t)$ versus time (Experiment).

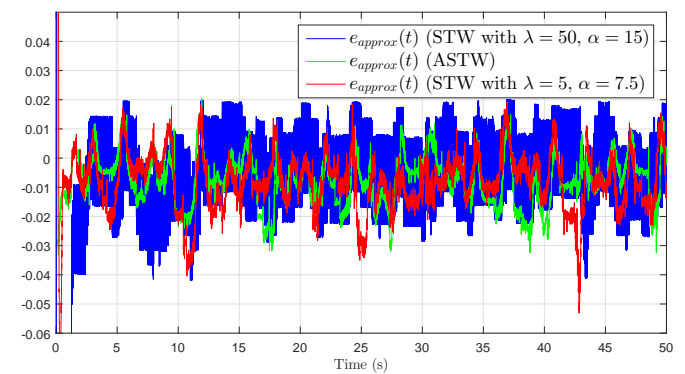

(c) Estimation error $e(t)$ of [16] and the proposed method (Experiment).

Fig. 4: Comparison between the proposed method and [16] with two different cases (Experiment).

the adaptive super-twisting algorithm, it is able to handle the tradeoff between the accuracy and the applicability.

\section{B. Simulation 1: TDE with channel inherent noise}

In this part, one takes into account the effect of the channel inherent noise [17] in the TDE problem. In this case, the accurate value of the delayed signal $s(t-h(t))$ is not available to the delay estimator, and the perturbed delayed signal

$$
s_{n}(t-h(t))=s(t-h(t))+n(t)
$$

with $n(t)$ a Gaussian noise ${ }^{1}$, is used to calculate the error term (11). Similar to [16, Section IV-E], one uses the $\mathscr{L}_{2}$ norm of the estimation error

$$
\|e\|_{\left[t_{1}, t_{2}\right]}=\left(\int_{t_{1}}^{t_{2}}|e(s)|^{2} \mathrm{~d} s\right)^{1 / 2}
$$

\footnotetext{
${ }^{1}$ As stated in [22, p.173], the channel inherent noise can be modeled as a Gaussian noise.
} 
to evaluate the TDE performance on $\left[t_{1}, t_{2}\right]$, lower $\|e\|_{\left[t_{1}, t_{2}\right]}$ represents more accurate estimation and less oscillation on $\left[t_{1}, t_{2}\right]$.

This simulation deals with the TDE of (23), the noise $n(t)$

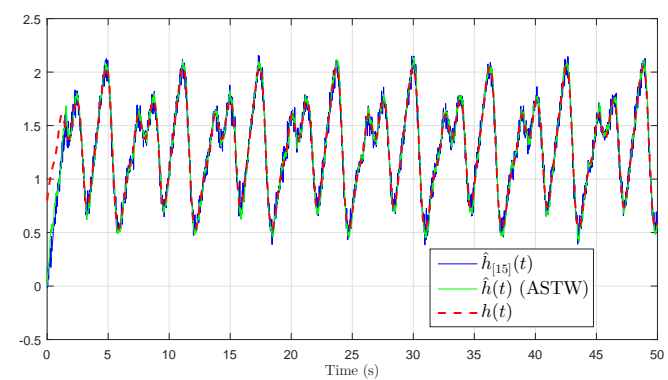

(a) Time-varying delay $h(t)$ and delay estimation $\hat{h}(t)$ with [15] and the proposed method (Simulation 1).

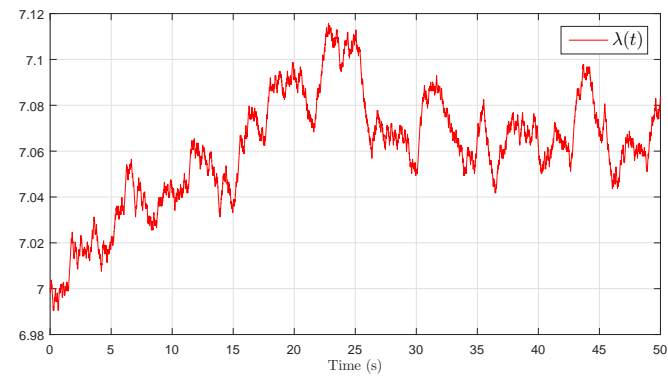

(b) Adaptive gain $\lambda(t)$ versus time (Simulation 1).

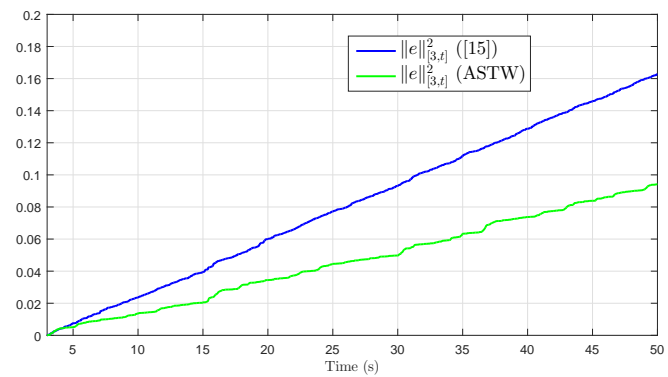

(c) $\mathscr{L}_{2}$ norms of the TDE error of [15] and the proposed method (Simulation 1).

Fig. 5: Comparison between the proposed method and [15] with channel inherent noise (26) (Simulation 1).

is chosen as a Gaussian noise such that $|n(t)| \leq 0.2$. The simulation results are presented in Figure 5. The parameters of the proposed method are the same as the ones given in section IV-A, except $\mu=0.04$ is redefined. Both methods can estimate the round-trip delay $h(t)$ by using the perturbed signal $s_{n}(t-h(t))$ with slight estimation error, but the channel inherent noise has lower effect on the proposed method (see Figure 5c). By comparing with the main theoretical results of [16], although the gains $\lambda$ and $\alpha$ are replaced by the adaptive version, the TDE algorithm is still robust with respect to the channel inherent noise.

\section{Simulation 2: TDE under deception attack}

In real application, the messages (measurement and control signal) of RCS can be transmitted via communication network. In this case, the adversary may hijack the communication network and inject false data into the measurement or control signal [20, Section VI-C] in order to damage the system. According to Remark 2, the proposed method is based on the communication between the plant and the controller, then it is at risk from the cyber attack. Thus, the main purpose of this simulation is to compare the performances of [15] and the proposed method under deception attack [23]. Consider the TDE problem given in (23), and one assumes that the communication network of $s(t)$ is under the following deception attack:

$$
s_{a}(t-h(t))=s(t-h(t))+\beta(t) \chi(t),
$$

with $\beta(t)$ a Bernoulli distribution such that

$$
\operatorname{Prob}\{\beta(t)=1\}=0.03, \quad \operatorname{Prob}\{\beta(t)=0\}=0.97 .
$$

The attack value $\chi(t)$ is set to:

$$
\chi(t)= \begin{cases}-0.3+\xi(t), & t \in[5,10] \cup[25,30] \cup[45,50] \\ 0.5+\xi(t), & t \in[15,20] \cup[35,40] \\ 0, & \text { otherwise }\end{cases}
$$

with $|\xi(t)| \leq 1$.

Remark 4: In real application, (28) is a sampled-data process with sampling period $T_{s}=0.005 \mathrm{~s}$, then (28) can be transformed into the discrete deception attack model [23, eq. (4)] such that:

$$
\operatorname{Prob}\{\beta(k)=1\}=0.03, \quad \operatorname{Prob}\{\beta(k)=0\}=0.97,
$$

and

$\chi(k)= \begin{cases}-0.3+\xi(k), & k \cdot T_{s} \in[5,10] \cup[25,30] \cup[45,50] \\ 0.5+\xi(k), & k \cdot T_{s} \in[15,20] \cup[35,40] \\ 0, & \text { otherwise }\end{cases}$

with $|\xi(k)| \leq 1$.

In this simulation, the proposed method stands on the following parameter setting: $\omega_{1}=\gamma_{1}=0.7, \mu=0.00025, \lambda_{m}=0.1$, $\eta=0.3, \zeta=0.8$, and $\lambda(0)=7$.

The simulation results are presented in Figure 6 . See Figure $6 \mathrm{a}$, when the communication network is not subject to deception attack (i.e. $t \in[0,5) \cup(10,15) \cup(20,25) \cup$ $(30,35) \cup(40,45])$, both methods can accurately estimate the round-trip delay $h(t)$. If the communication network is under the deception attack (28), the performance of [15] is more degraded than the proposed method. The estimation errors $e(t)$ of the two methods are displayed in Figure 6c, it is observed that [15] has large estimation error when the deception attack (28) appears, but the proposed method only has weak fluctuations during this period. Thus, the proposed method provides a better robustness than [15] when the delay estimation communication loop is under deception attack.

\section{CONCLUSION}

In this paper, one improves the TDE method introduced in [16] by using the adaptive super-twisting algorithm [18]. With this new technique, the gains of the TDE algorithm are 


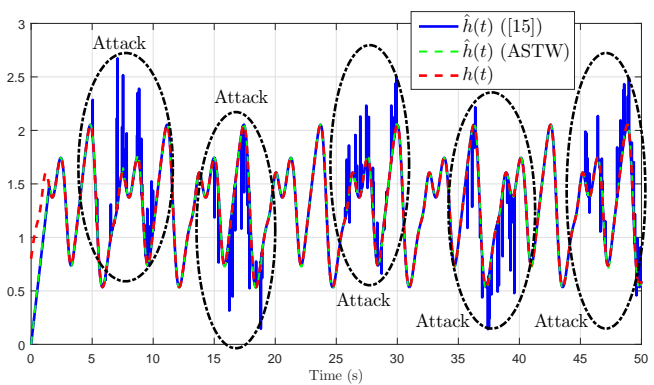

(a) Time-varying delay $h(t)$ and delay estimation $\hat{h}(t)$ with [15] and the proposed method (Simulation 2).

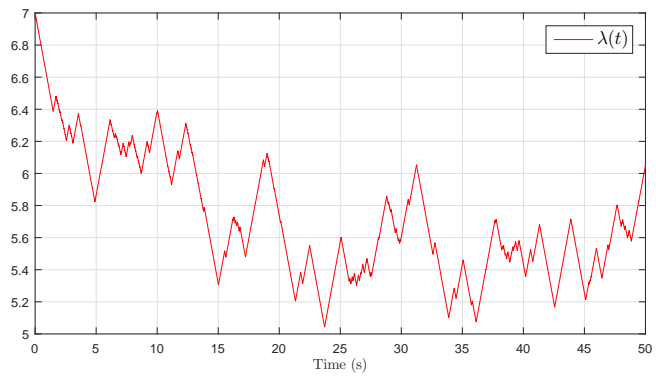

(b) Adaptive gain $\lambda(t)$ versus time (Simulation 2).

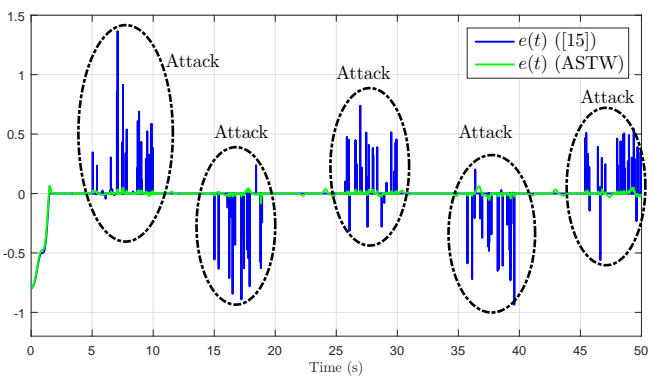

(c) Estimation error $e(t)$ of [15] and the proposed method (Simulation 2).

Fig. 6: Comparison between the proposed method and [15] under deception attack (28) (Simulation 2).

automatically tuned, and it is not necessary to pay attention to the parameter settings (14). The experiment and simulation results illustrate that the proposed method can achieve the expected TDE accuracy by using the lowest adaptive gains. Moreover, by comparing with the delay measurement method [15], the proposed method provides better robustness dealing with channel inherent noise and deception attack. The combination of the proposed method and the predictor-based controller [4] for real RCS will be investigated for future work.

\section{ACKNOWLEDGMENT}

The work of Yang Deng is supported by China Scholarship Council (CSC).

\section{REFERENCES}

[1] S.-H. Yang, Internet-based Control Systems: Design and Applications. New York, USA: Springer, 2011.

[2] E. Fridman, Introduction to time-delay systems: Analysis and control. New York, USA: Springer, 2014.
[3] D. Bresch-Pietri, J. Chauvin, and N. Petit, "Adaptive control scheme for uncertain time-delay systems," Automatica, vol. 48, no. 8, pp. 1536-1552, 2012.

[4] V. Léchappé, E. Moulay, and F. Plestan, "Prediction-based control of LTI systems with input and output time-varying delays," Systems \& Control Letters, vol. 112, pp. 24-30, 2018.

[5] X. Ren, A. B. Rad, P. Chan, and W. L. Lo, "Online identification of continuous-time systems with unknown time delay," IEEE Transactions on Automatic Control, vol. 50, no. 9, pp. 1418-1422, 2005.

[6] L. Belkoura, J.-P. Richard, and M. Fliess, "Parameters estimation of systems with delayed and structured entries," Automatica, vol. 45, no. 5, pp. 1117-1125, 2009.

[7] Y. Zhu, H. Su, and M. Krstic, "Adaptive backstepping control of uncertain linear systems under unknown actuator delay," Automatica, vol. 54, pp. 256-265, 2015.

[8] D. Etter and S. Stearns, "Adaptive estimation of time delays in sampled data systems," IEEE Transactions on Acoustics, Speech, and Signal Processing, vol. 29, no. 3, pp. 582-587, 1981.

[9] G. Zheng, A. Polyakov, and A. Levant, "Delay estimation via sliding mode for nonlinear time-delay systems," Automatica, vol. 89, pp. 266273, 2018.

[10] S. Diop, I. Kolmanovsky, P. Moraal, and M. Van Nieuwstadt, "Preserving stability/performance when facing an unknown time-delay," Control Engineering Practice, vol. 9, no. 12, pp. 1319-1325, 2001.

[11] S. V. Drakunov, W. Perruquetti, J.-P. Richard, and L. Belkoura, "Delay identification in time-delay systems using variable structure observers," Annual Reviews in Control, vol. 30, no. 2, pp. 143-158, 2006.

[12] Y. Tan, "Time-varying time-delay estimation for nonlinear systems using neural networks," International Journal of Applied Mathematics and Computer Science, vol. 14, pp. 63-68, 2004.

[13] X. Wu, Z. Sun, F. Liang, and C. Yu, "Online estimation of unknown delays and parameters in uncertain time delayed dynamical complex networks via adaptive observer," Nonlinear Dynamics, vol. 73, no. 3, pp. 1753-1768, 2013.

[14] L. Belkoura, "Identifiabilty of systems described by convolution equations," Automatica, vol. 41, no. 3, pp. 505-512, 2005.

[15] C.-L. Lai and P.-L. Hsu, "Design the remote control system with the time-delay estimator and the adaptive smith predictor," IEEE Transactions on Industrial Informatics, vol. 6, no. 1, pp. 73-80, 2010.

[16] Y. Deng, V. Léchappé, S. Rouquet, E. Moulay, and F. Plestan, "Super-twisting algorithm based time-varying delay estimation with external signal," IEEE Transactions on Industrial Electronics, 2019. [Online]. Available: http://dx.doi.org/10.1109/TIE.2019.2960739

[17] C. E. Shannon, "Communication in the presence of noise," Proceedings of the IEEE, vol. 86, no. 2, pp. 447-457, 1998.

[18] Y. Shtessel, M. Taleb, and F. Plestan, "A novel adaptive-gain supertwisting sliding mode controller: Methodology and application," Automatica, vol. 48, no. 5, pp. 759-769, 2012.

[19] Y. Shtessel, C. Edwards, L. Fridman, and A. Levant, Sliding mode control and observation. New York, USA: Springer, 2014.

[20] X.-M. Zhang, Q.-L. Han, X. Ge, D. Ding, L. Ding, D. Yue, and C. Peng, "Networked control systems: a survey of trends and techniques," IEEE/CAA Journal of Automatica Sinica, vol. 7, no. 1, pp. 1-17, 2019.

[21] T. M. Apostol, Mathematical analysis. Boston, USA: AddisonWesley, 1974.

[22] J. R. Pierce, An Introduction to Information Theory: Symbols, Signals \& Noise. New York, USA: Dover Publications, 1980.

[23] D. Ding, Z. Wang, D. W. Ho, and G. Wei, "Distributed recursive filtering for stochastic systems under uniform quantizations and deception attacks through sensor networks," Automatica, vol. 78, pp. 231-240, 2017 